Original Research Paper

\title{
Analytical Solutions of Riccati Fractional Equation with Coefficients Satisfying Differential Conditions with Arbitrary Function
}

\author{
Khaled K. Jaber \\ Department of Mathematics, Faculty of Science, Zarqa University, Zarqa, Jordan
}

\author{
Article history \\ Received: $15-10-2017$ \\ Revised: 07-12-2017 \\ Accepted: 26-12-2017 \\ Email: khaledjaber4@yahoo.com
}

\begin{abstract}
New exact solutions of the Fractional Riccati Differential equation are presented. Exact solutions are obtained in cases that the coefficient functions and an arbitrary function satisfy some differential conditions. In view of the conditions imposed on Riccati equation's coefficients we define and formulate the coefficients of the Riccati equation to obtain the differential cases, for each case the general solution is presented. The results obtained are useful and confirm the simplicity of the conformable definition of fractional derivative.
\end{abstract}

Keywords: Fractional Riccati Equation, Differential Conditions, Exact Solutions

\section{Introduction}

Integer order differential operator is a local operator, while fractional order operator is a non local operator. Mathematical modeling of systems in which the next state depends on the present state as well as the current state is better using fractional differential equations. Fractional calculus has many applications in physics, statistics, engineering and electrochemistry. Many phenomena in these fields modeled using differential equations of fractional order, especially the derivative with respect to the time (Kamke, 1959; Polyanin and Zaitsev, 2003; Soare et al., 2008; Mak and Harko, 2013; Cang et al., 2009; Tan and Abbasbandy, 2008; Abbasbandy, 2006a; Khalil et al., 2014; Aminikhah and Hemmatnezhad, 2010; Khan et al., 2011).

The general form of the linear first order differential equation can be written as $\frac{d y}{d x}=f(x, y)$. The function $f(x$, $y$ ) can be approximated as $f(x, y)=P(x)+Q(x) y+R(x) y^{2}$ $+\cdots$. If this approximation was terminated at the third term we get a non linear first order differential equation called Riccati Differential Equation (RDE) which is a type differ from all classical non linear first order differential equations. The first who deal with this kind of differential equations was the Italian mathematician Riccati (1676-1754). Riccati differential equation has many physical applications, such as particle motion under the effect of a central potential of power law representation and some applications in classical mechanics. Riccati differential equation was studied by many researchers (Kamke, 1959; Polyanin and Zaitsev, 2003; Soare et al., 2008). Mak and Harko (2013) studied the reduced Riccati equation for the integration of Navier Stokes and Schrodinger equations using new integrability case for Riccati equation with $Q(x)=0$.

The Fractional Riccati Differential Equation (FRDE) has the form:

$D^{a} y=P(x)+Q(x) y+R(x) y^{2}, x>0, \quad 0<a \leq 1$.

With the initial condition $y(0)=y_{0}$ and the coefficients $P(x), Q(x)$ and $R(x)$ are arbitrary continuous functions. Fractional Riccati equation was solved by many researchers using different methods, such as homotopy analysis method (Cang et al., 2009; Tan and Abbasbandy, 2008), homotopy perturbations method (Abbasbandy, 2006a; Aminikhah and Hemmatnezhad, 2010; Abbasbandy, 2006b), Adomian decomposition (Khan et al., 2011), Gulsu and Sezer (2006) gave a solution of the Riccati equation using the Taylor matrix method and many others.

In the conformable sense $D^{a} y \frac{d^{\infty} y}{d x^{\infty}}=x^{1-\infty} \frac{d y}{d x}$ so Equation (1) can be transformed to the form:

$y^{\prime}=p(x)+q(x) y+r(x) y^{2}, x>0$.

where, $p(x)=x^{\alpha^{-1}} P(x), q(x)=x^{\alpha^{-1}} Q(x), r(x)=x^{\alpha^{-1}} R(x)$. Using the substitution $y(x)=\frac{-u^{\prime}(x)}{u(x) r(x)} \operatorname{RDE}(2)$ can be 
transformed to the following differential equation $u^{\prime \prime}-\left[q(x)+\frac{r^{\prime}(x)}{r(x)}\right] u^{\prime}+p(x) r(x) u=0$, which is a second order differential equation. If a particular solution $y_{P}$ of Riccati differential equation is well known one can find its general solution, in the case that the particular solution is not known and its coefficient functions satisfy some conditions RDE could be integrated, otherwise, there is no chance to find an analytical solution. The classical method for solving Riccati differential equation depends on a given particular solution $y_{P}$. One can substitute $y(x)=y_{p}+\frac{1}{w}$ so, $y^{\prime}(x)=y_{p}^{\prime}-\frac{w^{\prime}}{w^{2}}$ into RDE (2) to be expressed as:

$$
y_{P}^{\prime}-\frac{w^{\prime}}{w^{2}}=P(x)+q(x) y_{P}+\frac{q(x)}{w}+r(x) y_{P}^{2}+\frac{r(x)}{w^{2}}+\frac{2 r(x) y_{P}}{w} .
$$

Since $y_{P}$ satisfies RDE (2), previous equation can be reduced to a first order linear differential equation of the form $w^{\prime}+\left[q(x)+2 r(x) y_{P}\right] w=-r(x)$ which can be solved by multiplying it by an integrating factor.

$$
\mu(x)=\exp \left(\int_{x_{0}}^{x}\left[q(t)+2 r(t) y_{P}(t)\right] d t\right), \text { then integrating to }
$$

get the following general solution $w=\frac{c-\int r(x) \mu(x) d x}{\mu(x)}$.

Using these results, FRDE (1) has the general solution:

$$
y=y_{P}+\frac{\exp \left(\int^{X} t^{\alpha-1}\left(Q(t)+R(t) y_{P}(t)\right)\right) d t}{C-\int r(x)\left\{\exp \left(\int^{X} t^{\propto-1}\left(Q(t)+R(t) y_{P}(t)\right)\right) d t\right\} d x} .
$$

The main object of this work is to construct appropriate conditions on the coefficient functions of fractional Riccati differential equation (1) using the approach used in (Harko et al., 2013).

\section{Preliminaries and Notations}

Fractional derivative and integration were defined by many famous mathematicians, such as Riemann, Liouville and Caputo. In this section, Riemann-Liouville and Caputo definitions will be given, in addition some basic properties of fractional calculus theory which are further used in this study.

\section{Definition 2.1.}

The Riemann-Liouville operator of fractional integral of order $a>0$, of a piecewise continuous function $f$ on [0, $\infty)$ such that it is integrable in any subinterval $(a, \infty)$ is defined as:

$$
{ }_{a} D_{t}^{a} f(t)=\frac{1}{\Gamma(n-a)}\left(\frac{d}{d t}\right)^{n} \int_{a}^{t} \frac{f(x)}{(t-x)^{a-n+1}} d x,(n-1) \leq \alpha<n
$$

where, $\alpha$ is any real number and $n$ is an integer.

\section{Definition 2.2.}

The Caputo fractional derivative of a function $f(t)$ is defined as:

$$
{ }_{a}^{c} D_{t}^{a} f(t)=\frac{1}{\Gamma(n-a)} \int_{a}^{t} \frac{f^{(n)}(x)}{(t-x)^{a+1-n}} d x,(n-1) \leq \alpha<n
$$

where, $\alpha$ is any real number and $n$ is an integer.

Khalil et al. (2014) gave a new definition of fractional derivative of order $\alpha \in(0,1]$. This definition is conformable to the definition of the derivatives of integer order, it is more natural and easier than previous definitions. Also, he generalized this definition for any $\alpha>0$. However, the case $\alpha \in(0,1]$ is the most important one and the other cases become easy when it is established.

\section{Definition 2.3.}

Conformable fractional derivative of order $\alpha \in(0$, 1] of a Given function $f:[0, \infty) \rightarrow \mathbb{R}$, is defined by (Khalil et al., 2014):

$$
T_{\alpha}(f)(t)=\lim _{\varepsilon \rightarrow 0} \frac{f\left(t+\varepsilon t^{1-a}\right)-f(t)}{\varepsilon}, \text { for all } t>0 .
$$

The Conformable fractional derivative at $t=0$ can be defined as $f^{(a)}(0)=\lim _{t \rightarrow 0+} f^{(a)}(t)$ provided that the limit exist and the function $f$ is $\alpha$-differentiable in some intervals $(0, a)$.

The conformable definition treats many shortages of previous definitions such as the derivative of the constant equals to zero, the product and fractional derivative rules are satisfied, also chain rule and anti-derivative rule are satisfied. In the present work, we adopting the Conformable Fractional Derivative definition.

\section{Solution Methods}

Fractional riccati equation was studied by many researchers. Some of these method will be discussed briefly In this section.

\section{Theorem 3.1. (Reduction to Second Order Equation)}

The non-linear fractional Riccati equation can be transformed using the substitution $v=y R(x)$, then the substitution $x^{1-a} v=-\frac{u^{\prime}}{u}$ into an ordinary differential equation of the form: 


$$
u^{\prime \prime}-\left(\frac{a-1}{x}+A(X)\right) u^{\prime}+X^{a-1} B(X) u=0
$$

where, $A(x)=x^{\propto-1} R(x)+\frac{p^{\prime}(x)}{P(x)}$ and $B(x)=x^{\propto-1} P(x) R(x)$. $P(x)$ is a non-zero and differentiable function, such that $\alpha \in(0,1]$, also the solution of this equation leads us to the solution:

$$
y=\frac{-u^{\prime}(x) x^{1-\alpha}}{P(x) U(x)} .
$$

\section{Theorem 3.2. (Transform FRDE to the Bernoulli} Equation)

For non-linear fractional Riccati equation the substitution $v(x)=y(x)-y_{P}(x)$ will transform the (FRDE) into the differential equation:

$$
v^{\prime}(x)+\left\{-2 x^{\propto-1} P(x) y_{P}-x^{\alpha-1} Q(x)\right\} v(x)=\propto x^{\propto-1} v^{2}(x),
$$

which is a Bernoulli equation with $n=2$ and $y_{P}$ is a known particular solution.

Theorem 3.3. (Obtaining Solution of FRDE by Abel's Formula)

The substitution $z=\frac{1}{y-y_{P}}$ in the FRDE (2), where $y_{P}$ is a particular solution of (2), will leads to the solution:

$$
z=e^{-1\left(2 P(x) y_{1}+Q(x)\right)} I_{a}\left(e^{I\left(2 P(x) y_{1}+Q(x)\right)}(-P(x))\right) .
$$

\section{Differentiability Conditions for Fractional Riccati Differential Equation}

Fractional Riccati differential equation (1) can be considered as an algebraic quadratic equation $R(x) y^{2}+$ $Q(x) y+\left(P(x)-D^{\alpha} y\right)=0$ in the variable $y$. The algebraic solution leads to its particular solution in the form:

$y_{P}=\frac{-Q(x) \mp \sqrt{Q^{2}(x)-4 R(x) P(x)+4 R(x) D^{\propto} y}}{2 R(x)}$

Previous equation of the particular solution along with an introduced generating function $f(x)$ that satisfies some conditions we will construct some differential conditions on the coefficients functions of the Fractional Riccati differential equation then give its general solution.

Recently, Harko et al. (2013) gave analytical solutions of Riccati equation, in their study they assumed that the functional coefficients of RDE satisfying some integral or differential conditions. Jaber and Tarawneh (2016) found an analytic solution of FRDE if its coefficients satisfy an integral condition. In this study we will construct more differential conditions on the coefficient functions involve arbitrary functions and give the general solution in each case.

Case 1:

$$
P(x)=\frac{1}{4 R(x)}\left[2 R(x) D^{\propto}\left(\frac{f_{1}}{R(x)}\right)-f_{1}^{2}-2 Q(x) f_{1}(x)\right] .
$$

Assume that the coefficient function $R(x)$ of the FRDE (2) satisfies the condition:

$$
y_{P}=\frac{f_{1}}{2 R(x)}
$$

where, $f_{1}(x) \in C^{\infty}(I)$ is an arbitrary function and $\mathrm{I}$ is an interval of real numbers. By equating the particular solution (5) of Equation (1) to the assumed form in Equation (6):

$$
\frac{-Q(x) \mp \sqrt{Q^{2}(x)-4 R(x) P(x)+4 R(x) \mathrm{D}^{\propto} y}}{2 R(x)}=\frac{f_{1}}{2 R(x)} .
$$

By some calculations it can be reduced to:

$$
Q^{2}(x)-4 R(x) P(x)+4 R(x) D^{\propto} \mathrm{y}=\left(f_{1}+Q(x)\right)^{2} .
$$

Performing the square and doing some rearranging to find an expression for $P(x)$ we get the first differentiability condition on coefficient functions of FRDE:

$$
P(x)-\frac{1}{4 R(x)}\left[2 R(x) D^{\propto}\left(\frac{f_{1}}{R(x)}\right)-f_{1}^{2}-2 Q(x) f_{1}(x)\right]
$$

In this case the FRDE (1) has the form:

$$
D^{\alpha} y=\frac{1}{4 R(x)}\left[\begin{array}{c}
2 R(x) D^{\alpha}\left(\frac{f_{1}}{R(x)}\right) \\
-f_{1}^{2}-2 Q(x) f_{1}(x)
\end{array}\right]+Q(x) y+R(x) y^{2} .
$$

Therefore the following theorem can be obtained:

\section{Theorem 4.1.}

If the coefficient functions of the FRDE (1) are chosen such that Equation (7) is satisfied, then the general solution of the FRDE (8) is given by:

$$
y=\frac{\exp \left(\int^{X} t^{\infty-1}\left(Q(t)+R(t) y_{P}(t)\right) d t\right.}{C_{1}-\int r(X)\left\{\exp \left(\int^{X} t^{\alpha-1}\left(Q(t)+R(t) y_{P}(t)\right) d t\right\} d x\right.}+\frac{f_{1}(x)}{2 R(x)} .
$$




\section{Case 2:}

$$
Q(x)=\frac{1}{f_{2}(x)}\left[R(x) D^{\propto}\left(\frac{f_{2}}{R(x)}\right)-2 R(x) P(x)\right]-\frac{f_{2}}{2} .
$$

Assume that the arbitrary function $f_{2}(x)$ and the coefficient functions satisfy the condition:

$$
y_{P}=\frac{f_{2}}{2 R(x)}
$$

where, $f_{2}(x) \in C^{\infty}$ (I) and $I$ is an interval of real numbers. By equating the particular solution (5) of Equation (1) to the assumed form in Equation (9):

$$
\frac{-Q(x) \pm \sqrt{Q^{2}(x)-4 R(x) P(x)+4 R(x) D^{\infty} y}}{2 R(x)}=\frac{f_{2}}{2 R(x)} .
$$

By some calculations it can be reduced to:

$$
Q^{2}(x)-4 R(x) P(x)+4 R(x) D^{\propto} y=\left(f_{2}+Q(x)\right)^{2} .
$$

Performing the square and doing some rearranging to find an expression for $P(x)$ we get the first differentiability condition on coefficient functions of FRDE:

$$
Q^{2}(x) \frac{1}{f_{2}(x)}\left[R(x) D^{\propto}\left(\frac{f_{2}}{R(x)}\right)-2 R(x) P(x)\right]-\frac{f_{2}(x)}{2}
$$

In this case the FRDE (1) has the form:

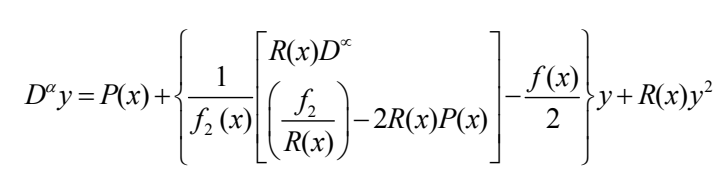

Therefore the following theorem can be obtained:

\section{Theorem 4.2.}

If the coefficient functions of the FRDE (1) are chosen such that equation (10) is satisfied, then the general solution of the FRDE (11) is given by:

$$
y=\frac{\exp \left(\int^{X} t^{\alpha-1}\left(Q(t)+R(t) y_{P}(t)\right) d t\right.}{C_{1}-\int r(x)\left\{\exp \left(\int^{X} t^{\alpha-1}\left(Q(t)+R(t) y_{P}(t)\right) d t\right\} d x\right.}+\frac{f_{2}(x)}{2 R(x)}
$$

Case 3:

$$
P(x)=\frac{Q^{2}(x)-4 R^{2}(x) f_{3}^{2}(x)}{4 R(x)}+D^{\propto}\left(\frac{-Q(X)}{2 R(x)} \pm f_{3}(x)\right) .
$$

Assume that the arbitrary function $f_{3}(x)$ and the coefficient functions satisfy the condition:

$y_{P}=\frac{-Q(X)}{2 R(x)} \pm f_{3}(x)$

where, $f_{3}(x) \in C^{\infty}(I)$ and $I$ is an interval of real numbers. By equating the particular solution (5) of Equation (1) to the assumed form in Equation (12):

$$
\frac{-Q(x) \mp \sqrt{Q^{2}(x)-4 R(x) P(x)}+4 R(x) D^{\alpha}\left(\frac{-Q(X)}{2 R(x)} \pm f_{3}(x)\right)}{2 R(x)}=\frac{-Q(X)}{2 R(x)} \pm f_{3}(x) .
$$

By some calculations it can be reduced to:

$$
4 R^{2}(x) f_{3}^{2}(x)=Q^{2}(x)-4 R(x) P(x)+4 R(x) D^{\propto}\left(\frac{-Q(X)}{2 R(x)} \pm f_{3}(x)\right) .
$$

By some calculations, we can find a differentiability condition on the coefficient functions of FRDE given as the following third condition:

$$
P(x)=\frac{Q^{2}(x)-4 R^{2}(x) f_{3}^{2}(x)}{4 R(x)}+D^{\propto}\left(\frac{-Q(X)}{2 R(x)} \pm f_{3}(x)\right) .
$$

In this case the FRDE (1) has the form:

$$
\begin{aligned}
& D^{\alpha} y=\frac{Q^{2}(x)-4 R^{2}(x) f_{3}^{2}(x)}{4 R(x)} \\
& +D^{\propto}\left(\frac{-Q(x)}{2 R(x)} \pm f_{3}(x)\right)+Q(x) y+R(x) y^{2} .
\end{aligned}
$$

Therefore the following theorem can be obtained.

\section{Theorem 4.3.}

If the coefficient functions of the FRDE (1) are chosen such that Equation (13) is satisfied, then the general solution of the FRDE (14) is given by:

$$
\begin{aligned}
& y=\frac{\exp \left(\int^{X} t^{\alpha-1}\left(Q(t)+R(t) y_{P}(t)\right) d t\right.}{C_{1}-\int r(x)\left\{\exp \left(\int^{X} t^{\alpha-1}\left(Q(t)+R(t) y_{P}(t)\right) d t\right\} d x\right.} \\
& -\frac{Q(x)}{2 R(x)}+f_{3}(x) .
\end{aligned}
$$

\section{Conclusion}

In the present paper we found exact solutions for the fractional Riccati differential equation using the conformable fractional derivative, by extending the work of (Harko et al., 2013) to fractional case. 


\section{Acknowledgments}

The author gratefully acknowledges that this research was supported by Dean scientific research in Zarqa University.

Also, the author would like to acknowledge the reviewers and the Editor of the journal for valuable suggestions.

\section{Ethics}

The author hereby certifies that This article is original. The material presented in this study can possibly be used to break public encryption keys. The author approved the manuscript and there are no ethical issues involved.

\section{References}

Abbasbandy, S., 2006a. Homotopy perturbation method for quadratic Riccati differential equation and comparison with Adomian's decomposition method. Applied Math. Computat., 172: 485-490. DOI: 10.1016/j.amc.2005.02.014

Abbasbandy, S., 2006b. Iterated He's homotopy perturbation method for quadratic Riccati differential equation. Applied Math. Computation, 175: 581-589. DOI: 10.1016/j.amc.2005.07.035

Aminikhah, H. and M. Hemmatnezhad, 2010. An efficient method for quadratic Riccati differential equation. Commun. Nonlinear Sci. Numerical Simulation, 15: 835-839. DOI: 10.1016/j.cnsns.2009.05.009

Cang, J., Y. Tan, H. Xu and S.J. Liao, 2009. Series solutions of non-linear Riccati differential equations with fractional order. Chaos, Solitons Fractals, 40: 1-9. DOI: 10.1016/j.chaos.2007.04.018

Gulsu, M. and M. Sezer, 2006. On the solution of the Riccati equation by the Taylor matrix method. Applied Math. Computat., 176: 414-421.

DOI: 10.1016/j.amc.2005.09.030
Harko, T., F. Lobo and M. K. Mak, 2013. Analytical solutions of the Riccati equation with coefficients satisfying integral or differential conditions with arbitrary functions. Universal J. Applied Math., 2: 109-118. DOI: 10.13189/ujam.2014.020206

Jaber, K.K. and S. Tarawneh, 2016. Exact solution of Riccati fractional differential equation. Universal J. Applied Math., 4: 51-54. DOI: 10.13189 /ujam.2016.040302

Kamke, E., 1959. Differential Gleichungen: Losungs Methoden Und Losungen. 1st Edn., Chelsea, New York.

Khalil, R., M. AlHorani, A. Yousef and M. Sababheh, 2014. A new definition of fractional derivative. J. Computational Applied Math., 264: 65-70.

Khan, N.A., A. Ara and M. Jamil, 2011. An efficient approach for solving the Riccati equation with fractional orders. Comput. Math. Applic., 61: 2683-2689. DOI: 10.1016/j.camwa.2011.03.017

Mak, M.K. and T. Harko, 2013. New further integrability cases for the Riccati equation. Applied Math. Computations 219: 7465-7471.

DOI: 10.1016/j.amc.2013.01.033

Polyanin, A.D. and V.F. Zaitsev, 2003. Handbook of Exact Solutions for Ordinary Differential Equations. 1st Edn., Chapman and Hall/CRC, Boca Raton.

Soare, M.V., P.P. Teodorescu and I. Toma, 2008. Ordinary Differential Equations with applications to Mechanics. 1st Edn., Springer Netherlands, Dordrecht, ISBN-10: 904817368X, pp: 488.

Tan, Y. and S. Abbasbandy, 2008. Homotopy analysis method for quadratic Riccati differential equation. Commun. Nonlinear Sci. Numerical Simul., 13: 539-546. DOI: 10.1016/j.cnsns.2006.06.006 